\title{
Comparing Chewable and Manual Toothbrushes for Reducing Dental Plaque: A Pilot Study
}

\author{
Moon-Jin Jeong, Hye-Sun Shin ${ }^{1}$, Soon-Jeong Jeong ${ }^{2}$, and Do-Seon $\mathrm{Lim}^{1 \dagger}$ \\ Department of Oral Histology and Biology, School of Dentistry, Chosun University, Gwangju 61452, \\ ${ }^{1}$ Department of Dental Hygiene, College of Health Science, Eulji University, Seongnam 13135, \\ ${ }^{2}$ Department of Dental Hygiene, College of Health Science, Youngsan University, Yangsan 50510, Korea
}

\begin{abstract}
This study aimed to compare the effectiveness of chewable toothbrush and manual toothbrush and provide basic data for recommendation of the chewable toothbrush in specific groups and situations. A total of 20 subjects participated in this study (rolling method, 10; non-rolling method, 10). After professional prophylaxis, participants used the manual toothbrush to brush their teeth for 3 minutes. After a 7-day wash-out period, participants used the chewable toothbrush according to the manufacturer's instructions. Pre- and post-plaque indexing of the teeth was performed. The dental plaque index was assessed using the Turesky Modification of the Quigley-Hein Plaque Index (TMQHPI) for amount of plaque and Silness-Löe Plaque Index (SLPI) for plaque thickness. The difference between pre- and post-dental plaque index was analyzed using a paired t-test and the Wilcoxon signed-rank test. The Mann-Whitney $U$ test was also used to compare the dental plaque index reduction rates. The dental plaque index differed significantly between the chewable toothbrush and the manual toothbrush. The TMQHPI reduction rate was significantly different between the rolling and non-rolling method groups for the manual toothbrush but not the chewable toothbrush. The difference in SLPI reduction rate between the rolling and non-rolling method groups was significant for the manual toothbrush but not for the chewable toothbrush. Differences in the dental plaque index reduction rates between the chewable and manual toothbrushes were not significant in the non-rolling method group. The results of this study showed higher reduction rates in dental plaque with manual toothbrush use than with chewable toothbrush use. However, the non-rolling method group did not show statistically significant differences according to toothbrush type. The present study showed that a chewable toothbrush can be an alternative to a manual toothbrush for individuals who have difficulty using the generally recommended rolling method.
\end{abstract}

Key Words: Chewable toothbrush, Dental care device, Dental plaque index

\section{Introduction}

Tooth brushing is reportedly the easiest and most cost-effective method of removing dental plaque and preventing dental caries and gingivitis ${ }^{1-3)}$. A recent meta-analysis by Zimmermann et al. ${ }^{4)}$ reported that a low frequency of tooth brushing increased the risk for periodontitis. The frequency of tooth brushing is also reportedly associated with diabetes ${ }^{5)}$, ardiovascular disease $^{6,7)}$, bone density ${ }^{8)}$, metabolic syndrome ${ }^{9)}$, and rheumatoid arthritis ${ }^{10}$. Since findings of the effect of tooth brushing have been reported, it is consistently argued that tooth brushing affects systemic health. Although tooth brushing is a fundamental daily dental care behavior, dental plaque is not effectively managed in many cases due to the wrong choice of toothbrush or toothpaste for an individual's dental health or an improper brushing technique. Accordingly, even if individuals are educated about tooth brushing techniques, a one-time educational session is not expected to have a positive effect ${ }^{11)}$. Furthermore, if the generally recommended rolling method is difficult for people with physical or other disabilities, people of a specific group, or those in certain situations, dental plaque should be managed in other ways. 
A chewable toothbrush is an oral hygiene product that was developed and commercialized in England. Developed as an alternative to a manual toothbrush, it consists of an elastic part that compresses when squeezed by the upper and lower jaws, a brush used for brushing the upper teeth in combination with the upper surface of the elastic part, and another brush that is used to brush the lower teeth in combination with the lower surface of the elastic part.

We reviewed the literature on chewable toothbrushes inside and outside of Korea and found only two articles that reported the ability of chewable toothbrushes to reduce dental plaque. Myoken et al. ${ }^{12)}$ showed a positive effect of chewable toothbrushes on reducing dental plaque in elderly Japanese patients, while Tugba et al. ${ }^{13)}$ reported in a study of 10- to 12-year-old children that the effect of a chewable toothbrush was the same as that of a manual toothbrush for reducing dental plaque. Accordingly, in this pilot study, we aimed to examine general adults, who are rarely studied in this research area, and assess the dental plaque indices before and after the use of a chewable or manual toothbrush to evaluate the reduction rates in dental plaque amounts and thicknesses to provide basic useful data for future recommendations for chewable toothbrush use.

\section{Materials and Methods}

\section{Study participants}

The study was approved by the Eulji University Institutional Review Board (EU15-27). Prior to participating, all participants were provided details of the study and provided written consent form.

The participants were conveniently sampled from the male and female students of the department of dental hygiene of a university located in Seongnam, Korea. The sample size was determined to be 20 , the minimum required to test a statistically significant difference between the two toothbrush types, and 10 each were selected for the group of individuals using the rolling tooth brushing method and the group of individuals not using the rolling method. As in the previous study ${ }^{13)}$, no participant in the present study had a systemic disease or wore an orthodontic appliance, and all had relatively good dental health without any serious periodontal disease.

\section{Procedure}

\section{1) Design}

The Fuzzy Brush ${ }^{\circledR}$ chewable toothbrush (Fuzzy Brush Ltd., Lancashire, United Kingdom) approved by the US Food and Drug Administration (Fig. 1) was used. The Fuzzy Brush ${ }^{\circledR}$ chewable toothbrush is similar in size to a manual toothbrush; contains xylitol, aroma mint, and an aquatic component; does not need toothpaste or rinsing; and is disposable after a single use. The participants were trained to roll the chewable toothbrush inside the mouth continuously for 3 minutes as if chewing gum according to the manufacturer's instructions. For the manual toothbrush, participants were instructed to brush the teeth for 3 minutes using a disposable Olight $\mathrm{Kouni}^{\mathrm{R}}$ toothbrush (Olight, Daegu, Korea) and Perio toothpaste ${ }^{\circledR}$ (LG Household \& Health Care, Seoul, Korea).

All participants $(n=20)$ received full-mouth debridement at the school lab. After a 2-week recovery period, each revisited the lab, were given a manual toothbrush, and instructed to brush the teeth for 3 minutes three times daily for 2 days ${ }^{13)}$. Afterward, they had a 7-day wash-out period during which they were instructed to brush their teeth using their own toothbrush. On the next visit to the laboratory after the wash-out period, they were given a

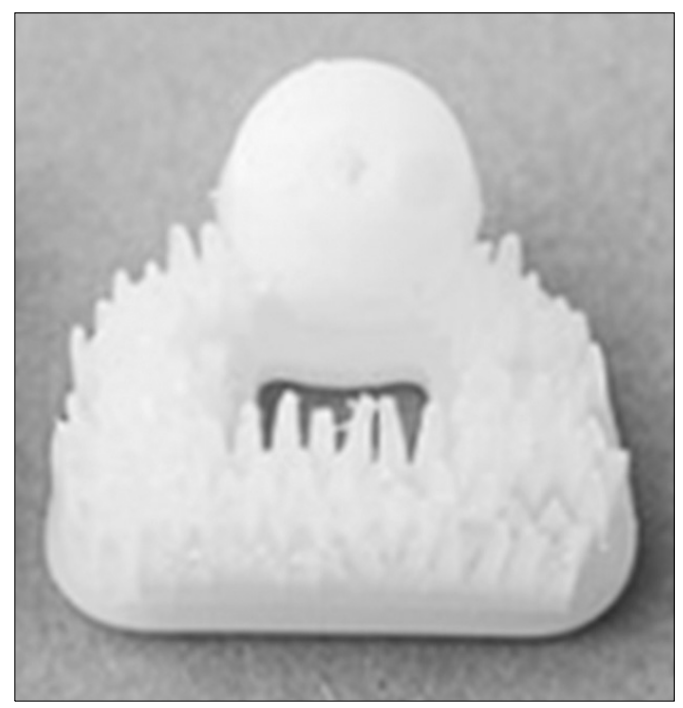

Fig. 1. Chewable brush. 
chewable toothbrush and instructed to use it instead of a manual toothbrush for 3 minutes three times daily for 2 days.

\section{2) Assessment of dental plaque indices}

Prior to each use of the chewable and manual toothbrushes, a disclosing solution was used to assess dental plaque amount and thickness. The dental plaque indices were re-assessed following the uses of chewable and manual toothbrushes.

First, the dental plaque amount was measured using the Turesky Modification of the Quigley-Hein Plaque Index (TMQHPI) ${ }^{14)}$. The TMQHPI scores were recorded as follows: 0 , dental plaque was not present; 1 , isolated areas of dental plaque; 2 , band of dental plaque $<1 \mathrm{~mm} ; 3$, dental plaque covered up to $1 / 3$ of the tooth surface; 4 , dental plaque covered between $1 / 3$ and $2 / 3$ of the tooth surface; and 5, dental plaque covered more than $2 / 3$ of the tooth surface.

Second, the dental plaque thickness was measured using the Silness-Löe Plaque Index (SLPI) ${ }^{15)}$. The SLPI score was recorded as follows: 0, dental plaque was not present; 1, a film of dental plaque adheres to the proximal surface and gingival margin of the tooth and is visible with the naked eye after the use of a disclosing solution or confirmed with an explorer; 2, a moderate amount of dental plaque adheres to the tooth surface, gingival margin, or gingival crevice; and 3, a large amount of dental plaque adheres to the gingival margin or crevice.

The TMQHPI was obtained for the overall area of a tooth by assessing six surfaces of each tooth (labial, mesiolabial, distolabial, lingual, mesiolingual, and distolingual). The TMQHPI obtained by adding the mesiolabial, mesiolingual, distolabial, and distolingual surface scores was used to specifically evaluate the effect of reduced dental plaque on the proximal surfaces. The SLPI was obtained for a total of six teeth (tooth numbers 12, 16, $24,31,36$, and 44) via assessment of the buccal and lingual surfaces.

To ensure reliable testing, 2 weeks before the experiment, a researcher took intraoral pictures of some of the participants using a digital camera and examined the tooth surface areas colored by the disclosing solution. The researchers then discussed how to make assessments according to the criteria of the two dental plaque indices and tried to attain high consistency in the evaluation of dental plaque indices via iterative training.

\section{Data analysis}

The Shapiro-Wilk test was performed to test normality of the pre- and post-tooth brushing dental plaque indices. The parametric analysis was conducted if a normality assumption was met, while a nonparametric analysis was conducted if the assumption was not met.

Comparisons of the dental plaque indices between preand post-use of chewable and manual toothbrushes were performed by testing the means and standard deviations using a paired t-test and the Wilcoxon signed rank test. For inter-group comparisons of dental plaque reduction rates, the Mann-Whitney U test was conducted of the chewable and manual toothbrushes and of the groups using the rolling and non-rolling methods. The statistical analyses were conducted using PASW Statistics ver. 18.0 program (IBM Co., Armonk, NY, USA).

\section{Results}

\section{Comparison of pre- and post-tooth-brushing dental} plaque indices with chewable and manual toothbrushes across all subjects

Table 1 shows the results of the analysis comparing the pre- and post-use of chewable and manual toothbrushes across all participants. Both in TMQHPI, which assesses the dental plaque amount, and in SLPI, which assesses the dental plaque thickness, the index scores were greatly reduced after versus before tooth brushing, and the difference was statistically significant in both indices. Particularly in SLPI, dental plaque reduction effects were similar in the conditions in which chewable versus manual toothbrush was used.

\section{Comparison of dental plaque reduction rates after use of chewable versus manual toothbrush condi- tions across all subjects}

Table 2 shows the results of the analysis conducted across all participants to compare dental plaque reduction 
Table 1. Mean of Plaque Index according to the Type of Toothbrush $(n=20)$

\begin{tabular}{lccccc}
\hline \multicolumn{1}{c}{ Type of toothbrush } & $\mathrm{n}$ & Before & After & Plaque reduction & $\mathrm{p}$-value* \\
\hline Manual toothbrush & & & & & \\
TMQHI & 20 & $3.03 \pm 0.50$ & $2.39 \pm 0.53$ & $0.64 \pm 3.60$ & $<0.001^{\mathrm{a}}$ \\
TMQHI-proximal & 20 & $3.12 \pm 0.54$ & $2.50 \pm 0.55$ & $0.62 \pm 0.32$ & $<0.001^{\mathrm{a}}$ \\
$\quad$ SLPI & 20 & $1.24 \pm 0.36$ & $1.06 \pm 0.24$ & $0.18 \pm 0.26$ & $0.001^{\mathrm{b}}$ \\
Chewable toothbrush & & & & \\
TMQHI & 20 & $3.01 \pm 0.52$ & $2.70 \pm 0.61$ & $0.31 \pm 0.41$ & $0.003^{\mathrm{a}}$ \\
TMQHI-proximal & 20 & $3.09 \pm 0.56$ & $2.76 \pm 0.60$ & $0.33 \pm 0.42$ & $<0.001^{\mathrm{b}}$ \\
SLPI & 20 & $1.23 \pm 0.35$ & $1.13 \pm 0.31$ & $0.10 \pm 0.12$ & $0.001^{\mathrm{a}}$ \\
\hline
\end{tabular}

Values are presented as mean \pm standard deviation.

TMQHI: Turesky Modified Quigley-Hein index, SLPI: Silness-Löe Plaque Index.

*Statistical significance $(\mathrm{p}<0.05)$.

${ }^{\mathrm{a}}$ Obtained from paired t-test, ${ }^{\mathrm{b}}$ obtained from Wilcoxon signed rank test.

Table 2. Comparison of Plaque Reduction Rate by the Type of Toothbrush

\begin{tabular}{lcccc}
\hline \multirow{2}{*}{ Plaque index } & $\mathrm{n}$ & \multicolumn{2}{c}{ Type of toothbrush } & p-value $^{\mathrm{a}}$ \\
\cline { 3 - 4 } & & Manual & Chewable & $0.002^{*}$ \\
TMQHI & 20 & $21.32 \pm 11.32(26.35)$ & $10.28 \pm 12.93(14.65)$ & $0.001^{*}$ \\
TMQHI-proximal & 20 & $20.16 \pm 10.22(26.85)$ & $10.48 \pm 12.86(14.15)$ & 0.251 \\
SLPI & 20 & $12.45 \pm 13.16(22.55)$ & $7.49 \pm 8.26(18.45)$ & \\
\hline
\end{tabular}

Values are presented as mean \pm standard deviation (mean rank) of plaque reduction rate.

TMQHI: Turesky Modified Quigley-Hein index, SLPI: Silness-Löe Plaque Index.

*Statistical significance $(\mathrm{p}<0.05)$.

${ }^{\mathrm{a}}$ Obtained from Mann-Whitney U test.

rates in the conditions in which a chewable versus a manual toothbrush was used. The reduction rate in dental plaque amount in the overall tooth area as well as the proximal surfaces was approximately twice higher in the manual toothbrush condition compared to the chewable toothbrush condition, and the differences were statistically significant $(p=0.002$ and $p=0.001)$. In contrast, dental plaque thickness reduction rates did not show a statistically significant difference between chewable and manual toothbrushes $(p=0.251)$.

\section{Comparison of dental plaque reduction rates after use of chewable versus manual toothbrush groups according to rolling brushing technique}

Focusing only on the group of individuals using the rolling method, the reduction rate in dental plaque amount showed a difference of approximately $15 \%$ between chewing and manual toothbrushes that was statistically significant $(\mathrm{p}=0.006$ and $\mathrm{p}=0.003)$. However, the reduction rate in dental plaque thickness was not significantly different $(p=0.060)$. For the group of individuals using a non-rolling method, the reduction rate in dental plaque amount showed a difference of approximately $7 \%$ between chewable and manual toothbrushes, which was not significant $(\mathrm{p}=0.131$ and $\mathrm{p}=0.096)$. The reduction rate in dental plaque thickness, $2 \%$, did not show a significant difference $(p=0.732$; Table 3$)$.

\section{Comparison of dental plaque reduction rates of the groups using rolling versus non-rolling method according to the type of toothbrush}

In the manual toothbrush condition, the reduction rate of dental plaque amount showed a significant difference, approximately $12 \%$, between groups using the rolling or non-rolling methods ( $p=0.023$ and $p=0.007$ ), while the difference in the reduction effects on dental plaque thickness was not significant $(\mathrm{p}=0.757)$. In the chewable toothbrush condition, the reduction rate of dental plaque amount did not show a significant difference between groups using rolling or non-rolling method in the overall 
Table 3. Comparison of Plaque Reduction Rate by Type of Toothbrush

\begin{tabular}{|c|c|c|c|c|}
\hline \multirow{2}{*}{ Plaque index } & \multirow{2}{*}{$\mathrm{n}$} & \multicolumn{2}{|c|}{ Type of toothbrush } & \multirow{2}{*}{ p-value ${ }^{a}$} \\
\hline & & Manual & Chewable & \\
\hline \multicolumn{5}{|l|}{ Rolling method } \\
\hline TMQHI & 10 & $27.39 \pm 10.30(14.10)$ & $12.51 \pm 17.09(6.90)$ & $0.006^{*}$ \\
\hline TMQHI-proximal & 10 & $26.10 \pm 9.67(14.40)$ & $12.65 \pm 16.91(6.60)$ & $0.003 *$ \\
\hline SLPI & 10 & $10.43 \pm 9.70(12.70)$ & $2.77 \pm 6.52(8.30)$ & 0.060 \\
\hline \multicolumn{5}{|l|}{ Non-rolling method } \\
\hline TMQHI & 10 & $15.25 \pm 9.76(12.50)$ & $8.04 \pm 7.05(8.50)$ & 0.131 \\
\hline TMQHI-proximal & 10 & $14.22 \pm 6.99(12.70)$ & $8.31 \pm 7.27(8.30)$ & 0.096 \\
\hline SLPI & 10 & $14.47 \pm 16.19(10.05)$ & $12.22 \pm 7.21(10.95)$ & 0.732 \\
\hline
\end{tabular}

Values are presented as mean \pm standard deviation (mean rank) of plaque reduction rate.

TMQHI: Turesky Modified Quigley-Hein index, SLPI: Silness-Löe Plaque Index.

*Statistical significance $(\mathrm{p}<0.05)$.

${ }^{\text {a } O b t a i n e d ~ f r o m ~ M a n n-W h i t n e y ~ U ~ t e s t . ~}$

Table 4. Comparison of Plaque Reduction Rate by Type of Tooth Brushing Method

\begin{tabular}{lcccc}
\hline Type of toothbrush & $\mathrm{n}$ & \multicolumn{2}{c}{ Method of tooth brushing $^{*}$ p-value $^{\mathrm{a}}$} \\
\cline { 3 - 4 } & & Rolling & Non-rolling & \\
Manual toothbrush & 10 & $27.39 \pm 10.30(13.50)$ & $15.25 \pm 9.76(7.50)$ & $0.023^{*}$ \\
TMQHI & 10 & $26.10 \pm 9.67(14.10)$ & $14.22 \pm 6.99(6.90)$ & $0.007^{*}$ \\
TMQHI-proximal & 10 & $10.43 \pm 9.72(10.10)$ & $14.47 \pm 16.19(10.90)$ & 0.757 \\
SLPI & & & & \\
Chewable toothbrush & 10 & $12.51 \pm 17.29(11.0)$ & $8.04 \pm 7.05(10.0)$ & 0.705 \\
TMQHI & 10 & $12.65 \pm 16.91(10.80)$ & $8.31 \pm 7.27(10.20)$ & 0.820 \\
TMQHI-proximal & 10 & $2.77 \pm 6.52(7.05)$ & $12.22 \pm 7.21(13.95)$ & $0.006^{*}$ \\
SLPI & &
\end{tabular}

Values are presented as mean \pm standard deviation (mean rank) of plaque reduction rate.

TMQHI: Turesky Modified Quigley-Hein index, SLPI: Silness-Löe Plaque Index.

*Statistical significance $(\mathrm{p}<0.05)$.

${ }^{\mathrm{a}}$ Obtained from Mann-Whitney U test.

tooth area or on the proximal surfaces $(\mathrm{p}=0.705$ and $\mathrm{p}=0.820$ ), but a statistically significant difference was found in the reduction rate of dental plaque thickness $(p=0.006$; Table 4$)$.

\section{Discussion}

The present study included 20 general adults and evaluated the dental plaque reduction effect of chewable and manual toothbrushes. Reduction rates were examined with respect to dental plaque amount (measured by TMQHPI) and thickness (measured by SLPI) in the overall tooth area and on the proximal surfaces. The dental plaque reduction effect was compared between chewable and manual toothbrushes as well as between groups using rolling and non-rolling method to test the effect and practicality of chewable toothbrushes.

To date, many studies have reported on various dental hygiene products other than manual toothbrushes, such as high-pressure oral spray devices ${ }^{16}$, sonic electric toothbrushes ${ }^{17)}$, and essential oil mouth rinses ${ }^{18)}$. However, no study has been conducted on chewable toothbrush use in general adults. The chewable toothbrush used in the present study is a new type of toothbrush that can reduce dental plaque using a masticatory motion without the use of the hands, unlike manual toothbrushes.

The analysis performed on the dental plaque indices before versus after the use of a chewable or manual toothbrush across all subjects showed that dental plaque amount and thickness decreased. The dental plaque 
amount reduction rate showed a statistically significant difference between chewable and manual toothbrushes in the mean and median. However, thickness reduction rates did not show a statistically significant difference.

A separate analysis was also conducted of the rolling versus non-rolling method groups. In the former group, the reduction rate of dental plaque amount was significantly different between the chewing and manual toothbrushes; in the latter, the reduction effects were statistically insignificant for dental plaque amount and thickness. Regarding thickness reduction rate in particular, the average rankings were similar, suggesting that the effect was almost the same. In other words, for those not using the rolling method, the chewable toothbrush had a reduction effect similar to that of the manual toothbrush.

In the manual toothbrush condition, the reduction rate in dental plaque amount was significantly different between the group using the rolling method and the group using the non-rolling method, and a significant difference was observed on the proximal surfaces as well. However, there was no significant difference between methods with respect to dental plaque thickness reduction rates. That is, a manual toothbrush had a large effect on reducing dental plaque for those using the rolling method. In the chewable toothbrush condition, there was no significant difference between tooth brushing methods in dental plaque amount reduction rate, nor was there a significant difference on the proximal surfaces. That is, the chewable toothbrush showed a similar reduction effect regardless of whether the individual used the rolling method. We believe, however, that the reason why the reduction rate in dental plaque thickness was significantly different between the rolling and non-rolling methods was because the dental plaque prior to the use of a chewable toothbrush was thicker in the group using a non-rolling method than in the group using the rolling method as shown in the mean dental plaque reduction effect of 12.22 and 2.77 for users of the non-rolling method and those of the rolling method, respectively.

In both groups using the rolling and non-rolling method, the reduction effect in the amount of dental plaque was greater in the manual toothbrush condition than in the chewable toothbrush condition. However, the difference between chewable and manual toothbrushes was relatively smaller in the group using the non-rolling method. Therefore, it is difficult for chewable toothbrushes to completely replace manual toothbrushes for effectively managing dental plaque. However, as argued by Frandsen ${ }^{19)}$, how individuals brush their teeth considerably influences dental plaque removal. Accordingly, we believe that for disabled individuals who have difficulty managing their dental health by themselves and always require help from caregivers, the physically disabled and elderly with limited mobility who cannot gain a positive effect from tooth brushing education using a manual toothbrush, and those in specific situations in which a toothbrush and toothpaste are unavailable, a chewable toothbrush can replace a manual toothbrush in terms of reducing dental plaque. Because the participants of the present study were young and healthy adults, we cannot hastily conclude that chewable toothbrushes are useful for a specific population but can gauge their practicality from this pilot study conducted in preparation of a main study on chewable toothbrushes. In addition, regarding whether it is practical to provide dental health education using a chewable toothbrush as a new dental management product for the aforementioned specific groups of individuals who have difficulty using the rolling method and specific situations, studies should be conducted including diverse age groups and various populations.

Myoken et al. ${ }^{12)}$ and Tugba et al. ${ }^{13)}$ compared the dental plaque reduction effect of chewable and manual toothbrushes and found similar effects. In both studies, when a manual toothbrush was used, the dental plaque reduction effect was greater on the buccal surface than the lingual surface, while the effect was greater on the lingual surface than the buccal surface when a chewable toothbrush was used. It was inferred that the finding was due to the user of a chewable toothbrush subconsciously placing it on the lingual rather than buccal surface. In the present study, assessments were not made separately for each tooth surface; therefore, direct comparisons are impossible. Examinations of the dental plaque reduction effect for each tooth surface should be compared in future studies.

The use of dental health management devices such as dental floss and an interdental brush after brushing the 
teeth is generally recommended, which demonstrates that removing dental plaque from the proximal surfaces is also important $^{20)}$. In a study comparing various toothbrushes, Pelka et al. ${ }^{21)}$ argued that dental plaque should be assessed even on the proximal tooth surfaces. Accordingly, we assessed the dental plaque indices for the proximal surfaces. In all cases, the dental plaque reduction rate following the use of a manual toothbrush was slightly lower on the proximal tooth surfaces than on the overall tooth area, whereas the reduction rate following the use of a chewable toothbrush was slightly higher on the former than the latter. Thus, it seems that a chewable toothbrush reduces dental plaque on the proximal surfaces.

The present pilot study evaluated the dental plaque reduction effect of a chewable toothbrush in general adults, and a follow-up main study should be conducted with a larger sample and for a longer term to more effectively examine the effect. In addition, to assess the practicality of chewable toothbrushes in specific groups of individuals and specific situations, appropriate study subjects should be selected based on relevant evidence. Finally, an index with high reliability and validity should be used to measure dental plaque thickness.

The present study showed that a chewable toothbrush can be an alternative to a manual toothbrush for individuals who have difficulty using the generally recommended rolling method as well as those in specific situations. In the future, chewable toothbrushes can be used to manage dental health.

\section{Acknowledgements}

This study was supported by research fund from Chosun University, 2016.

\section{References}

1. Axelsson P, Lindhe J: Effect of controlled oral hygiene procedures on caries and periodontal disease in adults. J Clin Periodontol 5: 133-151, 1978.

2. Haffajee AD, Arguello EI, Ximenez-Fyvie LA, Socransky SS: Controlling the plaque biofilm. Int Dent J 53 Suppl 3: 191-199, 2003.
3. Briner WW: Plaque in relation to dental caries and periodontal disease. Int Dent J 21: 293-301, 1971.

4. Zimmermann H, Zimmermann N, Hagenfeld D, Veile A, Kim TS, Becher H: Is frequency of tooth brushing a risk factor for periodontitis? A systematic review and meta-analysis. Community Dent Oral Epidemiol 43: 116-127, 2015.

5. Aggarwal A, Panat SR: Oral health behavior and HbA1c in Indian adults with type 2 diabetes. J Oral Sci 54: 293-301, 2012.

6. Fujita M, Ueno K, Hata A: Lower frequency of daily teeth brushing is related to high prevalence of cardiovascular risk factors. Exp Biol Med (Maywood) 234: 387-394, 2009.

7. Kuwabara M, Motoki Y, Ichiura K, et al.: Association between toothbrushing and risk factors for cardiovascular disease: a large-scale, cross-sectional Japanese study. BMJ Open 6: e009870, 2016.

8. Kim HJ, Kim YH, Cho KH, et al.: Oral health behaviors and bone mineral density in South Korea: the 2008-2010 Korean national health and nutrition examination survey. $\mathrm{J}$ Bone Miner Metab 34: 225-233, 2016.

9. Kobayashi Y, Niu K, Guan L, et al.: Oral health behavior and metabolic syndrome and its components in adults. J Dent Res 91: 479-484, 2012.

10. Marotte $\mathrm{H}$ : Tooth-brushing: an impact on rheumatoid arthritis. Joint Bone Spine 83: 619-621, 2016.

11. Hoogstraten J, Moltzer G: Effects of dental health care instruction on knowledge, attitude, behavior and fear. Community Dent Oral Epidemiol 11: 278-282, 1983.

12. Myoken $\mathrm{Y}$, Yamane $\mathrm{Y}$, Myoken $\mathrm{Y}$, Nishida T: Plaque removal with an experimental chewable toothbrush and a control manual toothbrush in a care-dependent elderly population: a pilot study. J Clin Dent 16: 83-86, 2005.

13. Tugba B, Canan D, Nurhan Ö: How effective is a chewable brush in removing plaque in children? A pilot study. J Pediatr Dent 3: 41-45, 2015.

14. Turesky S, Gilmore ND, Glickman I: Reduced plaque formation by the chloromethyl analogue of victamine C. J Periodontol 41: 41-43, 1970.

15. Silness J, Loe H: Periodontal disease in pregnancy. II. Correlation between oral hygiene and periodontal condition. Acta Odontol Scand 22: 121-135, 1964.

16. Park K, Park WC, Bae KH, Jin BH, Baek DI: Comparing the efficacy of a high pressure spray oral hygiene appliance and a 
sonic vibration toothbrush in reducing dental plaque. J Korean Acad Oral Health 38: 71-76, 2014.

17. Singh G, Mehta DS, Chopra S, Khatri M: Comparison of sonic and ionic toothbrush in reduction in plaque and gingivitis. J Indian Soc Periodontol 15: 210-214, 2011.

18. Honk SC, Kang ST, Shin JH, Lim YK, Lee DY: Comparison of the effectiveness of essential oil mouthrinse and interdental brush in patients with fixed orthodontic appliances. J Korean Dent Assoc 48: 371-378, 2010.

19. Frandsen A: Mechanical oral hygiene practices. State of the science review. In: Löe H, Kleinman DV, ed. Dental plaque control measures and oral hygiene practices: proceedings from a state-of-the-science workshop. IRL Press, Oxford, pp.93-116, 1986.

20. Robinson E: A comparative evaluation of the scrub and bass methods of toothbrushing with flossing as an adjunct (in fifth and sixth graders). Am J Public Health 66: 1078-1081, 1976.

21. Pelka AK, Nagler T, Hopp I, Petschelt A, Pelka MA: Professional brushing study comparing the effectiveness of sonic brush heads with manual toothbrushes: a single blinded, randomized clinical trial. Clin Oral Investig 15: 451-460, 2011. 